\title{
On "Landscaping" and Influence of Empirical Studies
}

\author{
Frank Houdek
}

Experimentation is considered to be an important element in technology transfer. Empirical results on new approaches should help to persuade decision makers to apply these approaches in their environment. But to what degree are decisions for new approaches driven by empirical results at all? From a subjective point of view, decisions for new approaches seem often to neglect empirical results at all. What are the reasons for this? To analyze this situation, we first have to see that there are at least three dimensions that affect technology selection:

1. Point in time for selection of new approaches. Usually, industry is not waiting for new approaches to be suggested. Established processes are maintained and sometimes improved evolutionarily. The window of opportunity, i.e. the period of time when a company seeks for new approaches is usually comparably small. Often, this window opens in conjunction with improvement initiatives (e.g. a CMMI assessment and a follow up program to cure identified deficits). Pushing approaches outside that window might only help to increase awareness technology adoption is bound to these windows.

As a consequence, proposals for new approaches should be (1) available during that time window and (2) related to typical deficits identified during such assessments (to be provocative: no one cares about optimal reading techniques it itself, but a efficient set of technologies to improve the KPA quality management might be very welcome).

2. Subjective degree of relevance. Technology adoption is driven by two main factors: need for change and trust that proposed technology might help (i.e. the risk of the particular technology is comparably low).

3. Relevant empirical results. Let us assume that the amount of relevant empirical knowledge in software engineering is depicted as a world map, we would see that only limited areas have already been discovered yet. Many areas are still completely unknown. Unfortunately, there is even no such map yet and building such a map should be a major activity in the next year.

However, a number of criteria to identify promising trails that should be followed (i.e. questions that should be taken into account) can be already identified. Three examples are given below:

- Cost-Benefit trade-off analysis (not primarily looking for best technique, but for the cost-efficient one)

- Robustness: Likeliness that technique survives even under project pressure

- Required background: Can the technique be beneficial be used with, e.g. electrical or mechanical engineers? 